\title{
FERROMAGNETIC BEHAVIOR OF A SHORT PERIOD ANTIFERROMAGNETIC SUPERLATTICE
}

\author{
Z. WILAMOWSKI \\ Institute of Physics, Polish Academy of Sciences \\ Al. Lotników 32/46, 02-668 Warszawa, Poland \\ W. JANTSCH AND W. FASCHINGER \\ Institut für Experimentalphysik and Institut für Halbleiterphysik \\ Johannes Kepler Universität, 4040 Linz-Auhof, Austria
}

We present a study of short period MnTe/CdTe superlattices by magnetic resonance. The usually antiferromagnetic structure exhibits critical line broadening above the Néel temperature typical of antiferromagnetic ordering, but also some ferromagnetic properties at lower temperature. We suggest that the observed ferromagnetic-like moment originates from coupling of the moments of the recently proposed strong magnetic polarons.

PACS numbers: 71.35.+z, 75.30.Et, 75.70.Fr

Recently, in EPR experiments on periodic 2D antiferromagnetic structures consisting of alternating layers of CdTe and MnTe [1] we observed super-paramagnetic moments which we interpreted in terms of strong magnetic polarons (SMP), a new type of bound polaron [2], which, according to our suggestion, results from the local breakdown of antiferromagnetism due to the ferromagnetic double-exchange $d-s p-d$ interaction [3] in the neighborhood of an acceptor. This concept resolves also an older question: earlier, the observed easily saturable polarization of luminescence $[4,5]$ was attributed to the creation of a magnetic polaron by polarization of free carriers by local spins. The time constant for the "creation" of these polarons was observed, however, in the femtosecond range [6], although the spin-lattice relaxation in these compounds is longer by orders of magnitude [7]. Within the SMP model, this puzzle can be resolved: the polarization of the photoluminescence is caused by the persistent SMPs which act as spin selective recombination centers, and the kinetics is limited only by the very fast capture of free carriers [2]. The SMP-EPR, however, was observed only if the number $m$ of $\mathrm{MnTe}$ monolayers within one superlattice period does not exceed 4 , whereas the strong circular polarization of photoluminescence (PL) is found also in a much bigger class of the 2D structures. 
The results presented in this paper shed some light on the reasons why paramagnetic resonance due to the moments of the magnetic polarons cannot be observed for $m>4$. For a sample with $m=5$, we found a peculiar magnetic resonance which appears only if a magnetic field is applied during cooling the sample through the Néel temperature. The character of this resonance indicates long range ordering of the local polaron moments. Due to the coupling of the magnetic polarons to the surrounding spins of the antiferromagnetic layer, the polaron moment is no longer independent and (super)paramagnetic, but becomes part of a macroscopically ordered system.

The sample with $m=5$ is the only sample for which a ferromagnetic-like resonance has been observed. Among 15 samples of superthin $\mathrm{MnTe} / \mathrm{CdTe}$ superlattices, no other sample exhibited similar behavior. Nevertheless, the temperature dependence of the EPR signal clearly indicates that the signal originates from the antiferromagnetic multi-layer structure with its characteristic Néel temperature. Moreover, the discussed sample constitutes the border between samples exhibiting at low temperature the real paramagnetic signal and those exhibiting no EPR signal due to polarons. A few other samples show a polaron-like signal which appears after cooling the sample under magnetic field but, in contrast to the sample with $m=5$, which shows a single, strong resonance line, those samples exhibit several weak, remanent lines.

Experimental results for the sample with $m=5$ are shown in Fig. 1. No resonance is observed if the sample is cooled down without any external magnetic field, $H_{\mathrm{c}}$. If the sample is cooled under magnetic field, the magnetic resonance appears, characterized by a single line with a $g$-factor higher than 2 . The peak-to-peak line amplitude, $A_{\mathrm{pp}}$, depends on the magnitude of the external field applied during cooling, $H_{\mathrm{c}}$, while the integrated intensity (equal to $A_{\mathrm{pp}} \Delta H^{2}$, where $\Delta H$ is the linewidth) is constant. The decay of the resonance amplitude is thus caused by line broadening only. In Fig. 1a, the linewidth of the magnetic resonance is shown. With decreasing $H_{\mathrm{c}}$ the linewidth increases and the amplitude decays, tending to zero at $H_{\mathrm{c}} \approx 2 \mathrm{kG}$. The critical behavior close to the critical field of $2 \mathrm{kG}$ is clearly evident. For lower $H_{\mathrm{c}}$ only a very weak residual resonance can be detected.

After cooling down under high magnetic field to $5 \mathrm{~K}$, the line is observed at a resonance field of $2 \mathrm{kG}$. The corresponding $g$-factor is 3.2 . When the sample is cooled at a lower field, the resonance field slightly decreases. These results are shown in the inset to Fig. 1a.

The temperature dependences of the resonance field and the linewidth, measured during a slow warming process, are shown in Figs. 1b and 1c, respectively. With increasing temperature, the line shifts to higher resonance fields. Around $60 \mathrm{~K}$, a critical behavior is seen: the resonance field increases sharply below $60 \mathrm{~K}$, followed by a constant line position (with $g=2$ ) at higher temperatures. This temperature value fits the Néel temperature expected for 2D MnTe layers [8]. The antiferromagnetic ordering below $60 \mathrm{~K}$ is corroborated by the temperature dependence of the linewidth at temperatures above $60 \mathrm{~K}$. Figure 1c indicates the critical line broadening, characteristic of the para-antiferromagnetic transition. 

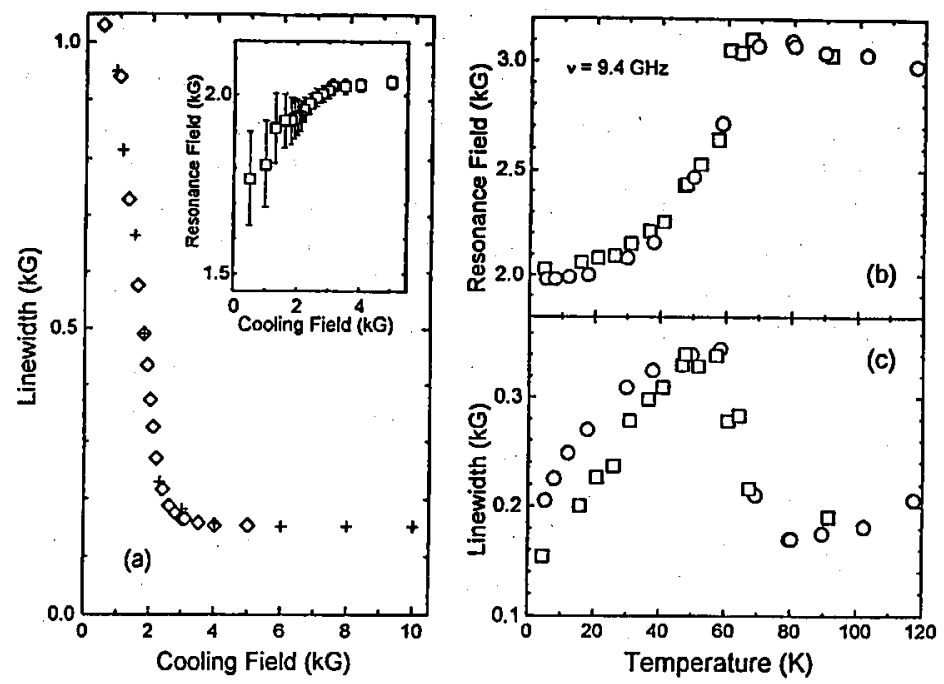

Fig. 1. EPR results for a ( $5 \mathrm{ML} \mathrm{MnTe}+8 \mathrm{ML} \mathrm{CdTe}) \times 100$ superlattice. (a) Dependence of the EPR peak-to-peak linewidth at $5 \mathrm{~K}$ on the cooling field for the magnetic field parallel (diamonds) and perpendicular (crosses) to the sample surface. The inset shows the dependence of the resonance field at $5 \mathrm{~K}$ on the cooling field. (b) Dependence of the resonance field on temperature. (c) Temperature dependence of the linewidth. The temperature dependences were measured during the warming process which followed the cooling down to $5 \mathrm{~K}$ under a field $4 \mathrm{kG}$ (squares) and $2.1 \mathrm{kG}$ (circles).

The temperature dependence of the integrated line intensity differs qualitatively from that observed for structures with $m<5$ which exhibit pure paramagnetic behavior, i.e., the EPR intensity is inversely proportional to the temperature. For the sample with $m=5$ the resonance intensity is, within experimental error of about $40 \%$, temperature independent, suggesting a saturation of the magnetic moment. This behavior is characteristic of a ferromagnetic resonance.

Below $60 \mathrm{~K}$ the line position, the linewidth, and simultaneously the line amplitude are influenced both by the cooling field and the temperature. The EPR spectra depend on the sample treatment prior to measurement, and in particular on the time the sample remains at intermediate temperatures. Moreover, the magnetic system of the investigated sample is not really persistent, and in the temperature range of $30-60 \mathrm{~K}$ a variation of the linewidth in time can be observed. These properties strongly indicate that at low magnetic field and low temperatures the magnetic moments tend to constitute a disordered system, similar to a spin glass. Apart from the mentioned spread of the characteristic times, also the following properties indicate the spin glass character: (i) the broadening of the resonance line, caused by fluctuations of the resonance frequency, which reflects the characteristic glass-like disorder and (ii) sensitivity of the low-field state on the external magnetic field. Apparently a field of $2 \mathrm{kG}$ is strong enough to order the system. 
Summarising, the experimental results show that the investigated short period $\mathrm{MnTe} / \mathrm{CdTe}$ superlattice exhibits unusual magnetic properties. They reflect antiferromagnetic, ferromagnetic, as well as spin glass properties. As a possible explanation of these puzzling data we suggest that the observed resonance originates from acceptor-bound SMPs. Only a small fraction of the Mn spins contributes to the magnetization of the polarons, but since the susceptibility, and thus the sensitivity of the magnetic resonance increases with the square of the magnitude of the individual spins, the spectrum of the mesoscopic moments of magnetic polarons becomes observable. For the super thin layers $(m<5)$, where the antiferromagnetic order is weak, and of short range, the super-paramagnetic behavior is observed. For a thicker layer $(m=5)$ the polaron spins are no more paramagnetic. Each of the mesoscopic moments is coupled, via short range $\mathrm{Mn}-\mathrm{Mn}$ coupling, to the long range ordered antiferromagnetic structure. This indirect coupling between the polarons is neither of ferro- nor of antiferromagnetic character. Instead it imposes rather a barrier which leads to the appearance of "viscosity" in the magnetic system, similar to that of spin glasses.

\section{Acknowledgments}

Herewith we would like to thank Heinz Krenn for many helpful discussions. This work is supported under a grant of the State Committee for Scientific Research (Republic of Poland) and by the Fonds zur Förderung der Wissenschaftlichen Forschung in Austria.

\section{References}

[1] W. Faschinger, Phys. Scr. Vol. T 49, 492 (1993).

[2] Z. Wilamowski, W. Jantsch, W. Faschinger, to be published.

[3] P.-G. de Gennes, Phys. Rev. 118, 141 (1960).

[4] M.R. Freeman, D.D. Awshalom, J.M. Hong, L.L. Chang, Phys. Rev. Lett. 64, 2430 (1990); M.R. Freeman, D.D. Awshalom, J. Appl. Phys. 67, 5102 (1990).

[5] A. Adleff, G. Hendorfer, W. Jantsch, W. Faschinger, Mater. Sci. Forum 143-147, 1408 (1994).

[6] D.D. Awshalom, M.R. Freeman, N. Samarth, H. Luo, J.K. Furdyna, Phys. Rev. Lett. 66, 1212 (1991).

[7] T. Strutz, A.M. Witowski, P. Wyder, Phys. Rev. Lett. 68, 3912 (1992).

[8] T.M. Giebultowicz, N. Samarth, H. Luo, J.K. Furdyna, P. Klosowski, J.J. Rhyne, Phys. Rev. B 46, 12076 (1992). 